\title{
Development and preliminary evaluation of a clinical guidance programme for the decision about prophylactic oophorectomy in women undergoing a hysterectomy
}

\author{
I Pell, J Dowie, A Clarke, A Kennedy, V Bhavnani
}

Qual Saf Health Care 2002;11:32-39

\begin{abstract}
Objectives: To develop a decision analysis based and computerised clinical guidance programme (CGP) that provides patient specific guidance on the decision whether or not to undergo a prophylactic oophorectomy to reduce the risk of subsequent ovarian cancer and to undertake a preliminary pilot and evaluation.

Subjects: Women who had already agreed to have a hysterectomy who otherwise had no ovarian pathology.

Setting: Oophorectomy decision consultation at the outpatient or pre-admission clinic.

Methods: A CGP was developed with advice from gynaecologists and patient groups, incorporating a set of Markov models within a decision analytical framework to evaluate the benefits of undergoing a prophylactic oophorectomy or not on the basis of quality adjusted life expectancy, life expectancy, and for varying durations of hormone replacement therapy. Sensitivity analysis and preliminary testing of the CGP were undertaken to compare its overall performance with established guidelines and practice. A small convenience sample of women invited to use the CGP were interviewed, the interviews were taped and transcribed, and a thematic analysis was undertaken. Results: The run time of the programme was 20 minutes, depending on the use of opt outs to default values. The CGP functioned well in preliminary testing. Women were able to use the programme and expressed overall satisfaction with it. Some had reservations about the computerised format and some were surprised at the specificity of the guidance given.

Conclusions: A CGP can be developed for a complex healthcare decision. It can give evidence-based health guidance which can be adjusted to account for individual risk factors and reflects a patient's own values and preferences concerning health outcomes. Future decision aids and support systems need to be developed and evaluated in a way which takes account of the variation in patients' preferences for inclusion in the decision making process.
\end{abstract}

See end of article for authors' affiliations Correspondence to: $\operatorname{Dr}$ A Clarke, Health Services Research Unit, London School of Hygiene \& Tropical Medicine, Keppel Street, London WC1E 7HT, UK;

aileen.clarke@|shtm.ac.uk

Accepted for publication 12 November 2001

$\mathrm{P}$

roviding individual patients with decision support to allow them to take a fuller part in treatment decisions-if and how they wish-is likely to become an increasingly important component to improving quality in health care. Patient involvement in decision making increases adherence to treatment choices and improves satisfaction and the appropriateness of interventions. ${ }^{12}$ It is clear that many patients both value and are able to make use of enhanced information about treatments and treatment choices. A variety of methods of delivering enhanced evidence-based patient information have accordingly been developed, including leaflets, decision boards, audiotapes, videotapes, interactive laser disks, and computer programs. ${ }^{3-5}$ Despite these developments, the presentation of research based information to patients, and especially recommendations, is still rare in practice and the practical difficulties involved are considerable.

Not unconnected with these difficulties is legitimate concern about the rate at which high quality research findings enter clinical practice, independent of any consideration of informing patients. Until now the main approach to disseminating best practice more effectively throughout the medical community has been the development of expert based guidelines. However, standard written guidelines cannot hope to cover the range of inputs and potential outcomes that need to be taken into account for a specific patient, even when management of a common condition is the focus. Nor can they respond to individual patient preferences in relation to health outcomes in the rigorous way that is provided in relation to clinical evidence.
Our aim was to address these issues simultaneously through the development of a computerised clinical guidance programme (CGP) based on broad decision analytical principles which would integrate research based evidence (adjusted for individual patient risk factors) and patient preferences in order to arrive at an explicit recommendation. All the assumptions and evidence underlying the recommendation would be accessible on request. The prototype CGP described here has been developed in the context of prophylactic oophorectomy (PO).

\section{BACKGROUND}

\section{Prophylactic oophorectomy (PO)}

Women who have decided to undergo a hysterectomy face, in the absence of compelling clinical indications, a further decision as to whether or not to have concurrent PO. This is a surgical operation to remove the ovaries as a prophylactic procedure against ovarian cancer. The decision, although apparently straightforward, is a complex one. Expert opinions on the appropriateness of $\mathrm{PO}$ vary and there is considerable variation in practice. In the UK in 1989, 20\% of gynaecologists said they would routinely remove the ovaries of premenopausal women aged $45-49$ years in the course of hysterectomy and $51 \%$ said they would do so in the case of premenopausal women aged over 49 years. ${ }^{6}$ The annual incidence of ovarian cancer in the UK is $1 / 3800$ in women aged over 25 and $1 / 2500$ in those aged over 55 , with a lifetime risk estimated at $1.5-2 \%$. Estimates of the proportion of ovarian cancers potentially prevented by use 
of PO vary between $1 \%$ and $11 \% .^{78}$ The complexity of the decision whether or not to undergo PO lies in the relative rarity of ovarian cancer coupled with the multiple indirect effects of removing the ovaries. While surgical removal effectively precludes the development of ovarian cancer, it creates indirect effects on other body systems through the cessation of oestrogen production, with notable effects on the chances of developing breast cancer, osteoporosis and osteoporosis related fractures, and heart disease. Taking supplemental oestrogen in the form of hormone replacement therapy (HRT) can modify these indirect effects to some extent. The decision therefore depends on a number of factors relating to the individual clinical characteristics of the patient and her family history, on the values she attaches to a number of different disease states of varying severity, and on her views on and anticipated compliance with HRT. The patient is faced with the task of making a set of trade offs involving the direct and indirect effects of the decision. It is doubtful if the unaided patient or clinician is capable of making these complex trade offs intuitively. Certainly, in their survey of PO, Jacobs and Oram $^{6}$ found that most gynaecologists were using a simple age related rule. It did not appear that important competing outcomes or the influence of personal or family history were being taken into account in any explicit way. This finding, coupled with the evident complexity of the decision, suggests that modelling the decision may be one obvious solution.

\section{Decision analytical techniques}

Decision analytical techniques have typically been seen as a suitable basis for guideline and clinical policy development, and rarely envisaged as being directly applicable in the clinical situation to help clinician and patient working together to make the optimal decision for that individual. However, they are useful if patients and clinicians value analysis based conclusions about their treatment, systematic exploration of their relevant preferences, and if either the patient, doctor, or both would like instant access to research based information about their choices and possible outcomes. Decision analytical models provide the technology required, enabling the patient and clinician to examine all aspects of the decision explicitly and transparently. ${ }^{9}$

\section{METHODS}

\section{System design}

The clinical guidance programme (CGP) is based on a set of Markov cycle tree models ${ }^{10}$ which allow for net benefit or loss from PO to be calculated under varying durations of HRT. There is one Markov model for each of the four main outcomes affected by the PO decision: cardiovascular disease, breast cancer, osteoporosis related major fracture, and ovarian cancer.

A Markov model is designed so that a hypothetical patient population-all with the characteristics of the individual under consideration-is taken through a predetermined number of cycles (a year long in our case). Each individual is always in one of a set of exclusive states during a cycle. For example, in the cardiovascular disease model the component diagnostic states (defined as disease states that can be uniquely identified using the WHO ICD10 taxonomy) were "well", "angina", "myocardial infarction", and "dead". Whether an individual stays in his or her current state or switches to a different state is defined by an (evidence-based) transition probability-for example, the probability of a woman who is previously "well" developing angina. The experience of this hypothetical population as it progresses through a series of annual cycles gives us the expected outcome of an intervention for an individual, calculated as evidencebased life expectancy (LE) given the characteristics of the individual under consideration.
It should be noted that the CGP deals implicitly with multiple states whereby an individual with ovarian cancer might also have a hip fracture because the results of all four Markov models are used to derive the final figures for LE, but it is assumed that the condition specific transition probabilities are not affected by the occurrence of multiple states.

Apart from LE, the CGP also calculates the net benefit of (or loss from) oophorectomy as quality adjusted life expectancy (QALE). In order to undertake the "quality adjustment" of LE, each period of time spent in each diagnostic state (cycle) is multiplied by the utility of being in that state for each of the Markov models. We therefore needed to map each diagnostic state (for example, angina) on to a set of health states, those of the generic multidimensional index EQ-5D. ${ }^{11}{ }^{12}$ We found no usable empirical mappings for our diagnostic states and therefore used consensus estimates from 10 healthy volunteers (including doctors, postgraduate students, and researchers in health related fields). They were asked to use their awareness and personal experience to assess the percentage of time they considered a patient in each of the relevant diagnostic states would spend at a particular level on each of the EQ-5D dimensions (pain, mobility, etc). The CGP applies a woman's individual quality adjusted values, explicitly elicited by the program, to the relevant health states she will pass through (fig 1), thus ensuring that the QALE reflects her outcome preferences. If a woman finds the process of having her preferences elicited either too difficult or too time consuming, the model allows her to elect to use standard health state valuations derived from published tariffs resulting from a population survey. ${ }^{12}$

\section{Information requirements}

To perform the relevant calculations the CGP requires information regarding both clinical factors and patient preferences. This is obtained from the literature (incidence, mortality, and relative risks) and from the patient (age, personal risk factors, and attitude to risk).

\section{Evidence from the literature}

Evidence from the literature was obtained by systematic searching of Medline, BIDS/Embase, and the Cochrane Database of Systematic Reviews for each major outcome and key risk factors in relation to four interventions: hysterectomy with oophorectomy with and without subsequent HRT and hysterectomy alone with and without HRT. The search strategy was based around the retrieval of relevant systematic reviews with as high a level of sensitivity and precision as possible, using a variety of free text methodological terms. It was also designed to ensure that randomised controlled trials and large scale epidemiological observational studies were included. Major outcomes included were coronary heart disease, breast cancer, ovarian cancer, and osteoporosis. Risk factors included were family history, smoking, parity, age at menarche, and exercise levels. Back searching and hand searching of articles was undertaken to ensure that relevant material (systematic reviews or large scale randomised or observational studies) were included. Incidence and survival data for the major outcomes for women in the appropriate age groups were obtained from a number of sources, principally the Office of National Statistics, the US National Cancer Institute, the Surveillance, Epidemiology and End Results (SEER) database, the American Heart Association, and relevant previous analyses. ${ }^{13} 14$ Two authors (AC, AK) assessed studies for use. In order to be included, publications had to have relevant subjects, interventions, and outcomes. Data were gathered using a hierarchy of evidence (meta-analysis, systematic review, randomised controlled trial, prospective observational study). Evidence was collected for all four conditions and for each diagnostic state-for example, for coronary heart disease we gathered evidence on the effect of PO, HRT, and relevant risk factors on the incidence of angina and myocardial infarction. 


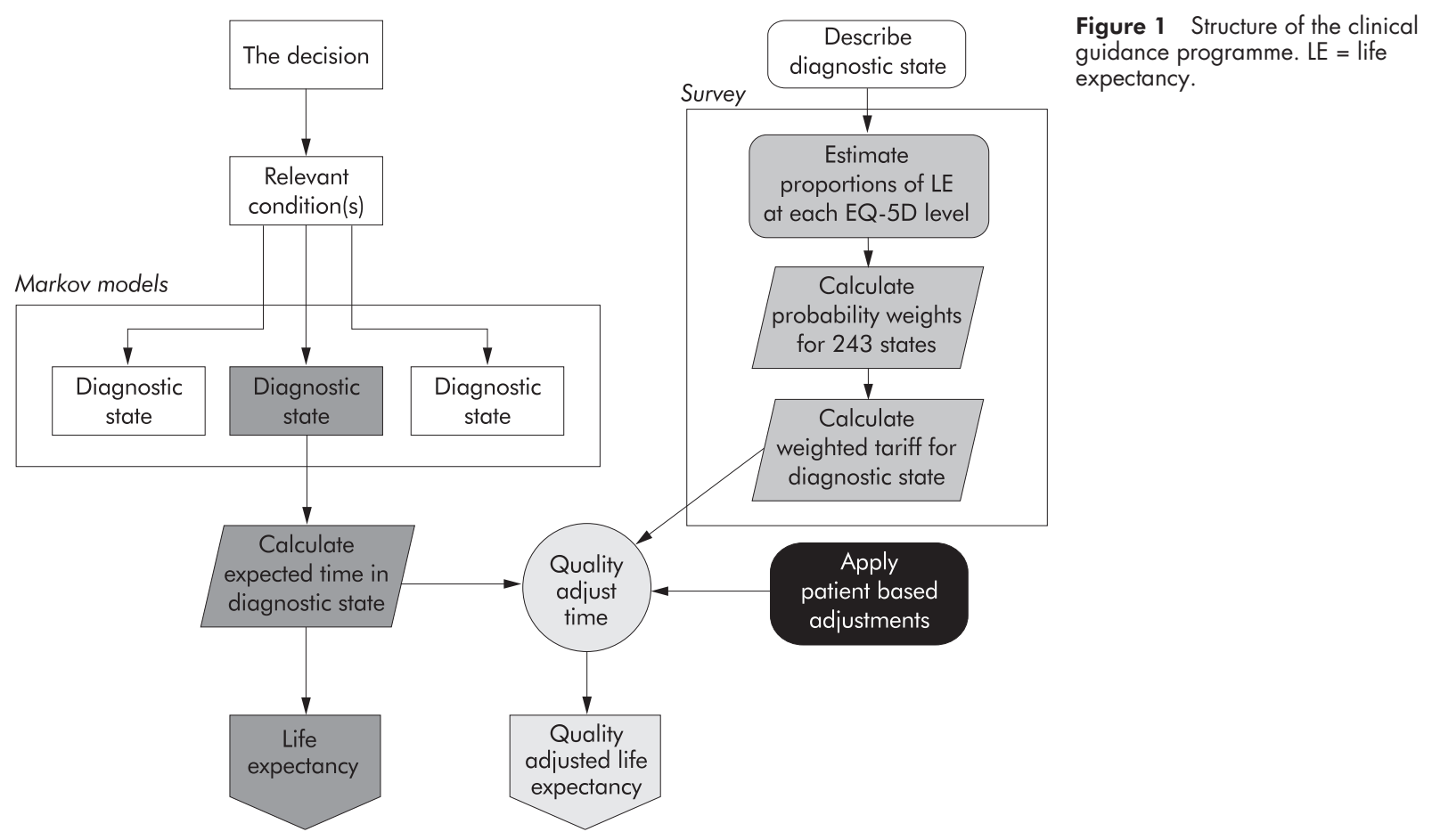

Patient information

Patient information was obtained during the running of the CGP in a number of stages. The patient was first asked for her name (or other unique identifier), age, and relevant risk factors such as smoking habits, family history of breast and ovarian cancer, or previous osteoporotic fracture. The various outcomes of the decision are heavily influenced by the hormonal status of the patient, whatever the decision on oophorectomy. Since this status is altered if the patient takes HRT, the patient was asked about her (provisional) intentions toward this therapy under each option. (Because the time period for taking HRT is self-selected, the model assumes $100 \%$ concordance with these intentions in its baseline result but a sensitivity analysis provides the patient with the results for a wide range of durations.) In order to individually tailor the output from the model, the patient was asked to indicate her risk preference (by a single health related standard gamble item) and to rate a number of health states using the standard "time trade off" method. In this step (and after explanation and a trial run), the woman was asked to say how many years of normal health she would be prepared to give up in order to be free of a particular combination of factors affecting her health such as reduced mobility and moderate pain. (Alternative configurations using the standard gamble and visual analogue scale approaches are built in but were not used in this pilot.)

At most stages the patient could opt out to default (population based) values if she wished, although she was always asked to confirm that she understood that the final results would potentially be less tailored to her own values than they would otherwise have been.

\section{Calculations}

For each of the four conditions (ovarian cancer, breast cancer, cardiovascular disease, and osteoporosis related major fracture) the number of years the patient is expected to spend in each specific diagnostic state under the oophorectomy option was calculated and added to produce LE values for each condition. For quality adjustment, the individual times spent in a diagnostic state were multiplied by the patient's values for that diagnostic state and added to produce individualised QALE measures for each condition. This process was repeated using the same set of patient values and risk factors, but this time adjusting the probabilities to reflect the expected consequences of the no oophorectomy option. The difference between the results for the two interventions was presented in the form of a guidance statement (fig 2). This always favours the option with the higher average QALE, irrespective of the size of the difference. The results were presented to three decimal places to allow for small differences such as a few "quality adjusted life weeks" to be shown.

All the numbers underlying the various results are available in a series of increasingly detailed screens if requested by the patient or her physician. A sensitivity graph then allows the woman to see the effect of any change from her provisionally expressed intention in relation to HRT. Finally, the programme produces a full report of the consultation, which may be printed out for the patient or physician to take away and study. If requested, a pictorial representation of the gains and losses by condition can also be printed (fig 3). The report is automatically updated if the patient returns to the programme to revise any of her inputs, and the programme can be rerun with changed inputs if the patient so wishes.

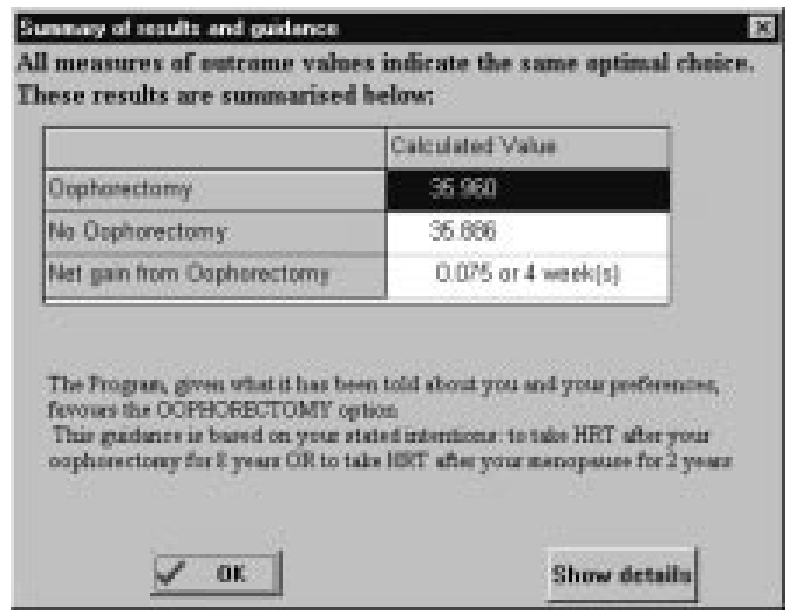

Figure 2 Summary results screen. 


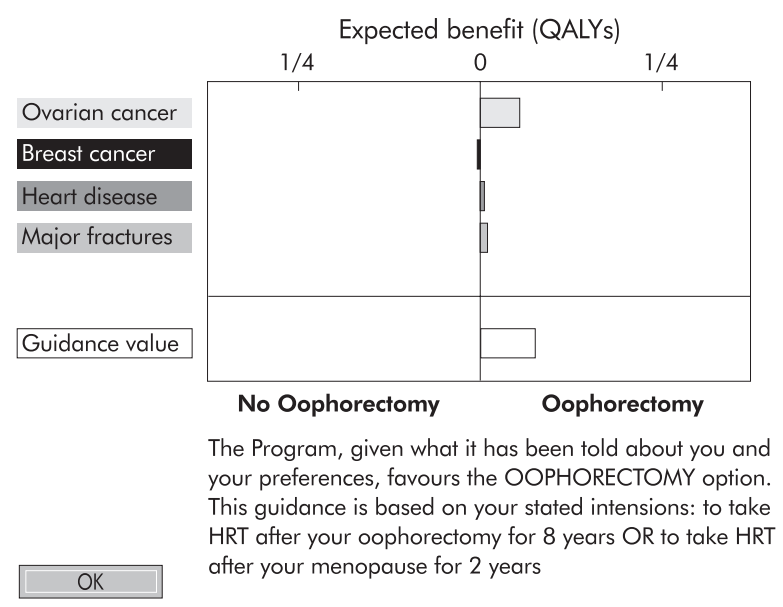

Figure 3 Condition comparison screen. This summary screen shows the net benefits for a particular individual in undergoing oophorectomy. Several points should be noted from the figure: (1) benefits occur in three of the four major outcome conditions; (2) the risk of ovarian cancer is reduced if the patient undergoes oophorectomy; (3) as stated in the text, the patient has indicated previously in the CGP that she will take hormone replacement therapy (HRT) and the benefits of this are evident in reduction in the risk of heart disease and major fractures; (4) the patient's risk of breast cancer is almost unchanged, but this is the net effect of a combination of a reduction in risk due to oophorectomy coupled with an increase in risk due to HRT; $(5)$ the overall expected benefits are small, although the output suggests a definite decision.

\section{Patient and clinician input into the design process}

Women in the appropriate age range from three community groups in southern England and the Midlands (two hysterectomy support groups and one non-health related group) were invited and agreed to help in the design process. Focus groups were held with each group before the CGP was designed to elicit key topic areas of importance for inclusion. The focus groups were taped and transcribed and a thematic analysis was undertaken. Preliminary versions of the CGP were piloted with each group and comments were noted in detail. A number of amendments were made to the programme in response to these comments. The CGP was also demonstrated at a number of meetings with gynaecologists in the study hospitals, their comments were noted, and amendments were made to the CGP in response.

\section{Preliminary testing of the CGP}

A sensitivity analysis was undertaken, varying assumptions about the effects of quality adjustment, and the anticipated guidance from the CGP was compared with published guidelines and with actual practice in the UK using a prospective dataset of 16000 women undergoing hysterectomy in the UK in 1994/5 (A Metcalfe, K McPherson, personal communication).

\section{Preliminary piloting of the CGP}

Approval to administer the CGP and to undertake in-depth qualitative interviews was obtained from the NHS Executive London Regional Office (MREC) research ethics committee and from the local research ethics committees of six participating hospitals. A convenience sample of 19 women recruited from six hospitals in south east England consented to participate in the preliminary pilot study. All were about to undergo hysterectomy with the possibility of undergoing oophorectomy. Women were taken through the CGP with one of the researchers acting as a facilitator (VB) and 10 women then participated in semi-structured interviews. The interviews were transcribed and analysed independently by two researchers $(\mathrm{AC} / \mathrm{VB})$ and a thematic analysis was undertaken.

\section{RESULTS}

\section{Patient and clinician input to design process}

Patient and clinician input to the design process resulted in a number of changes to the CGP including revision of the screens which explain the "time trade off" task, changing of the word "hypertension" to "high blood pressure" throughout, and the addition of an evidence base viewer. A major change was addition of introductory screens with full explanatory text for each task. These could be switched on or off at the facilitator's discretion.

\section{Preliminary piloting}

All the women were able to use the CGP with a facilitator. During the preliminary pilots with patients the CGP took between 10 and 25 minutes to run the programme (mean run time 20 minutes) The run time depends largely on the extent to which individual values are entered or default options used.

Results of the qualitative analysis are summarised in table 1. Patients were grateful for the time and attention paid to their decision and overall satisfaction with the CGP was high. There was some mistrust of computers and computer programs and a feeling that a "human touch" was important. Some women found the "time trade off" exercise initially difficult although most understood what was required after being presented with one or two health states to value; some reported that they found it difficult to think about "abstract" health states.

The CGP is unusual in giving a very explicit guidance statement and this appeared to be problematic, especially for women who received advice which was discrepant with their previously held intentions. However, the CGP appeared to clarify the PO decision for most of the women.

\section{Sensitivity analysis}

By analysing the outputs from CGP simulation runs, the results for any combination of the current data inputs were established in the form of an equation (box 1).

Taking one extreme case (where all living diagnostic states other than "well" are valued at 0-that is, all quality adjustments at 0 ), the equation returns a net benefit of -0.523 QALE (about 27 quality adjusted weeks) so the patient interested only in disease free years should not undergo oophorectomy. Taking the other extreme (where all living diagnostic

Table 1 Summary of qualitative findings about clinical guidance programme

\begin{tabular}{ll}
\hline Expectations & Clarification of decisions \\
\hline Process of using decision aid & $\begin{array}{l}\text { Most tasks completed by most women. "Standard gamble" and "time trade off" exercises found most difficult because } \\
\text { of difficulty of thinking in the abstract }\end{array}$ \\
$\begin{array}{l}\text { Reactions to the output } \\
\text { Surprise at specificity of guidance }\end{array}$ \\
$\begin{array}{l}\text { Impact on decisions } \\
\text { pecititudes towards overall }\end{array}$ & $\begin{array}{l}\text { Overall satisfaction. Seen as offering "time to think and talk". Some negative attitudes to the technology apparent } \\
\text { presentation of decision aid }\end{array}$ \\
\hline
\end{tabular}


Box 1 Equation derived from the CGP describing the net benefit of oophorectomy

As an example, the equation for the net benefit of oophorectomy for a 40 year old woman with no clinical risk factors and planning to take no HRT whether or not she undergoes $\mathrm{PO}$ is as follows:

Net benefit $=0.457(\mathrm{CHSV})-(1.04 . \mathrm{U}(\mathrm{OC}))$

$+0.049(\mathrm{CHSV})-(0.025 . \mathrm{U}(\mathrm{BC}))$

$-0.252(\mathrm{CHSV})+(0.101 . \mathrm{U}(\mathrm{Ang}))+(0.014 . \mathrm{U}(\mathrm{MI}))$

- 0.777(CHSV) + (0.588.U(Spine)) + (0.022.U(Hip))

where CHSV = current health state valuation, $U=$ utility (e.g. in quality adjusted life years), $\mathrm{OC}=$ ovarian cancer, $\mathrm{BC}=$ breast cancer, Ang = angina, $\mathrm{Ml}=$ myocardial infarction, Spine $=$ vertebral fracture, $\mathrm{Hip}=$ hip fracture

states are valued at 100 - that is, all quality adjustments at 1.0 ), the net benefit is +0.073 QALE (about 4 quality adjusted weeks) so the patient who values living (in any state) equally (and rates them all as good as full health) should undergo oophorectomy. Between these hypothetical extremes the net benefit can be calculated for any set of utilities elicited from the patient.

\section{Comparison of CGP with previous analyses and published guidelines}

Speroff $e t a l^{13}$ studied the effects of non-compliance with prescribed HRT. They modelled four strategies (PO with and without HRT and natural menopause with and without HRT) and found that, while perfect compliance always favours oophorectomy, partial compliance changes the decision and the CGP agrees with the full model strategy given in Speroff's paper. We assumed 100\% concordance by women with their own estimates of the duration of time for which they anticipated taking HRT. However, we also included a graph in the CGP itself which shows women the effects of changing the duration of HRT and how sensitive the decision is (in their particular case) to HRT duration.

Grann et $a l^{15}$ studied a population of BRCAl or BRCA2 patients (who have a genetic predisposition to breast cancer) and the potential gain to them of PO and/or mastectomy. To this end they modelled breast and ovarian cancer in detail, but did not incorporate any of the collateral effects of oophorectomy, nor did they explicitly consider the effects of HRT. They estimated that PO increased LE for a 30 year old subject by 0.4-2.6 years. A comparison with our result for a patient who has one first degree relative with breast cancer shows that the CGP calculates an increase of 1.6 life years with PO. However, while this figure is right in the middle of the range reported by Grann et al, the methods of obtaining health state values were different and a comparison of absolute values is not possible.

Schrag et $a l^{16}$ also studied BRCA positive subjects and reported a gain in LE of 0.5-1.5 years, depending on the probability of breast cancer and ovarian cancer. This again compares with our figure of 1.4 years for a 30 year old subject. On the basis of these published studies, the CGP appears to provide guidance that is in line with, but not identical to, that which can be derived from other published decision analyses.

\section{Comparison with current practice and guidelines}

Between the ages of 42 and 46 the proportion of women who have both ovaries removed during hysterectomy rises from approximately $30 \%$ to $60 \%$ (fig 4 ). The net expected benefit of oophorectomy as calculated by the CGP was compared with the reported proportion of $\mathrm{PO}$ in practice derived from a major cohort study of women undergoing hysterectomy in the UK in 1994/5. A monotonic relationship between the two was found, but this was non-linear. A good fit was obtained by log transformation of the proportion of PO. Least squares linear

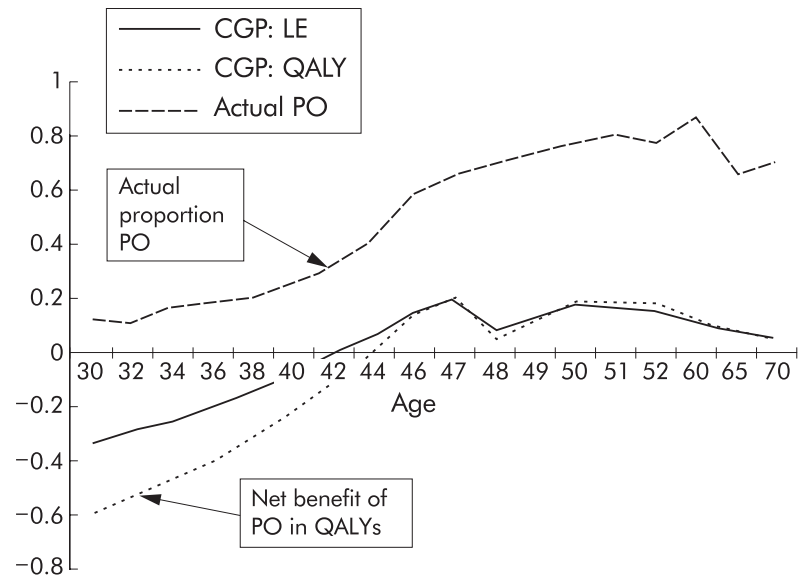

Figure 4 Comparison of benefit (in QALYs) from hysterectomies where oophorectomy is undertaken at different ages as predicted by the CGP and proportion of hysterectomies where oophorectomy was actually undertaken in the UK in 1995.

regression (net benefit versus $\log _{\mathrm{e}}$ observed proportion) gave an adjusted $r^{2}$ value of $0.953\left(\mathrm{~F} 6.17 \mathrm{e}^{-11}\right)$ for QALE. This result suggests that the guidance produced by the CGP is closely related to, but not synonymous with, current practice. Discrepancies occur at around the age of 46 years where the CGP suggests that the net benefit of PO tends to flatten off, although in actual practice the proportion of POs undertaken continues to rise.

The College of Physicians and Surgeons of Manitoba published clinical guidelines on this topic in $1999^{17}$ and the CGP's recommendations are also broadly in line with this guidance.

\section{DISCUSSION}

We have developed a clinical guidance programme which employs decision analytical techniques to model the consequences of undertaking PO or not during a total abdominal hysterectomy. The programme is based on research evidence and supplies guidance in the form of an explicit statement favouring one or other intervention, as well as detailed numerical results and graphical representations. It draws on the patient's own risk factors and preferences for health state outcomes. In this respect it is an innovation, combining individual values and preferences with the research evidence to produce individualised evidence-based guidance.

Piloting of the CGP showed that it is feasible to use in the clinical context and is valuable in clarifying the decision. The sensitivity analysis shows that quality adjustment is a valuable addition in that it has the potential to "swing" the decision one way or another. In none of our patients did the QALE based recommendation actually differ from that which would have been made by the LE result in terms of the sign of the difference, although the size of the difference did vary considerably in several cases.

The proportion of oophorectomies recommended by the CGP at a population level is reasonably in line with that produced by current practice. However, that should not necessarily be regarded as a valid test since the CGP can improve the appropriateness and quality of decisions at an individual level through its ability to take account both of the evidence on all relevant outcomes and of an individual's risk factors and values.

\section{Limitations of the study}

Why was a conventional decision tree not used? Decision trees require that the outcomes at any chance node should be mutually exclusive and collectively exhaustive. Thus, if the 
decision involves a number of non-exclusive outcomes, the classic decision tree format becomes practically unworkable. A further problem with the classic formulation is that it takes no account of time dependency in the probabilities. Converting the model to a Markov cycle tree helps to solve this latter problem, but this still requires that the transition probabilities from any state represent mutually exclusive and exhaustive transitions. One approach, used by Speroff et $a l^{13}$ in their earlier decision analysis of the oophorectomy decision, is to create a number of "combination states" and to model transitions explicitly between combinations. We took an alternative approach which seeks to cope with the exponential increase in complexity by a combination of decomposition of the problem into a number of diagnostic states and abstraction of these into health states.

There is considerable discussion as to the relative value of the "time trade off" approach which we used to establish women's individual diagnostic state valuations in order to obtain QALE values. ${ }^{18} 19$ The CGP allows for these values to be elicited by means of "time trade off", reference lottery, or visual analogue scale. Given that one of these must be chosen, we selected "time trade off" for use in the pilot study mainly because of the existence of "time trade off" based population values that could be used as defaults.

Following the work of Cher et $a l^{20}$ we included as a further configuration option a one off, conventionally phrased standard gamble item in the CGP that establishes the patient's risk preference. (The patient is asked at what level she would accept a treatment that has a chance of either returning her to perfect health or results in death compared with no treatment which would result in good health but for a shorter period of time.) The resulting parameter is used to risk adjust "time trade off" valuations before further calculation. The CGP reported here does not incorporate costs, but it could very simply be extended to incorporate a cost element and produce outcomes calculated in terms of cost per unit of quality adjusted time.

A drawback of many guidelines and decision aids is that the evidence base becomes outdated. One of the advantages of the data structure used in the CGP is that it can easily be updated to reflect new research findings. We see the programme as dynamic rather than as a static product.

The explicit guidance produced by the CGP is both a strength and a problem. The programme intentionally gives very specific guidance in line with the inputs provided (and its underlying assumptions), but it may cause problems if a patient or clinician already holds a strong intention or opinion. We need to undertake research to understand better the optimal timing for decision aids such as the CGP so that they can be used to their fullest potential as a readily available "third opinion". We see development of a more "user friendly" interface as important in ensuring that both clinicians and patients feel comfortable with the format.

\section{CONCLUSIONS}

We have developed a computerised clinical guidance programme based on decision analytical techniques which models the consequences of health interventions, uses both the available evidence and patient-specific information on risks and preferences to provide guidance, and which has been successfully piloted with a small number of patients. The decision to perform an oophorectomy on women between the ages of 40 and 50 is controversial. By making explicit the assumptions and evidence upon which its precise guidance is based, the CGP makes it possible for the physician and patient to explore in detail the trade offs and personal assumptions that will affect the decision. Transparent as far as both principles and data are concerned, the CGP can improve the quality and appropriateness of a decision and could be regarded by doctor and patient as an innovative and immediately available expert opinion. The reactions of individual women to the CGP in this

\section{Key messages}

- A clinical guidance programme (CGP) can be developed for a complex healthcare decision.

- The programme gives specific individualised evidencebased health guidance which is adjusted to account for individual risk factors and a patient's own values and preferences concerning health outcomes.

- In a preliminary pilot study women were able to use the CGP and expressed overall satisfaction with it.

- Decision aids and support systems need to be developed to take account of patients' preferences for inclusion in the decision making process.

pilot study suggest that work is required in future designs to ensure a more "user friendly" interface. It will also be important to take account of individual preferences for the extent and type of desired participation in decision making.

\section{ACKNOWLEDGEMENTS}

The authors acknowledge the help and cooperation of the Hysterectomy Support Groups who participated in the project and the individual women and gynaecologists who were involved in the development and piloting processes. The work was supported by a steering group for whose expert contribution we are grateful. We are sorry to report that unfortunately Ian Pell died suddenly in June 2001 shortly after completing this work.

\section{..................}

Authors' affiliations

I Pell, J Dowie, Environmental Epidemiology Unit, London School of Hygiene \& Tropical Medicine, London WC IE 7HT, UK

A Clarke, V Bhavnani, Health Services Research Unit, London School of Hygiene \& Tropical Medicine, London WCIE 7HT, UK

A Kennedy, Health Economics Research Group, Brunel University, London UB8 3PH, UK

Funding: This project was funded by the NHS Executive R\&D Implementation Programme and subsequently by the NHS R\&D Service Delivery and Organisation Programme.

Conflicts of interest: none.

\section{REFERENCES}

1 Dowie J. Evidence-based, cost-effective and preference-driven medicine: decision analysis based medical decision making is the pre-requisite. J Health Serv Res Policy 1996;1:104-13.

2 Dowie J. The research-practice gap and the role of decision analysis in closing it. Health Care Anal 1996;4:5-18

3 Liao L, Jollis JG, DeLong ER, et al. Impact of an interactive video on decision making of patients with ischemic heart disease. J Gen Intern Med 1996;11:373-6.

4 Shepperd S, Coulter A, Farmer A. Using interactive videos in general practice to inform patients about treatment choices: a pilot study. Fam Pract 1995; 12:443-7

5 Coulter A. Partnerships with patients: the pros and cons of shared clinical decision making. J Health Serv Res Policy 1997:2:112-21.

6 Jacobs I, Oram D. Prevention of ovarian cancer: a survey of the practice of prophylactic oophorectomy by fellows and members of the Royal College of Obstetricians and Gynaecologists. Br J Obstet Gynaecol 1989;96:510-5.

7 Grundsell H, Ekman G, Gullberg B, et al. Some aspects of prophylactic oophorectomy and ovarian carcinoma. Ann Chir Gynaecol $1981 ; 70: 36-42$.

8 Sightler SE, Boike GM, Estape RE, et al. Ovarian cancer in women with prior hysterectomy: a 14-year experience at the University of Miami. Obstet Gynecol 1991;78:681-4.

9 Dolan JAG. Can decision analysis adequately represent clinical problems? J Clin Epidemiol 1990;43:277-84.

10 Sonnenberg FA, Beck JR. Markov models in medical decision making: a practical guide. Med Decis Making 1993;13:322-38

11 EuroQol Group. EuroQol: a new facility for the measurement of health-related quality of life. Health Policy 1990;16:199-208.

12 Dolan P. Modeling valuations for EuroQol health states. Med Care 1997;35: 1095-108.

13 Speroff T, Dawson NV, Speroff L, et al. A risk-benefit analysis of elective bilateral oophorectomy: effect of changes in compliance with estrogen therapy on outcome. Am J Obstet Gynecol 1991;1 64:165-74. 
14 Todd CJ, Palmer C, Camilleri-Ferrante C, et al. Differences in mortality after fractures of the hip: the East Anglian Audit. BM 1995;310:904-8.

15 Grann VR, Panageas KS, Whang W, et al. Decision analysis of prophylactic mastectomy and oophorectomy in BRCA1-positive and BRCA2-positive patients. J Clin Oncol 1998;16:979-85.

16 Schrag D, Kuntz KM, Garber JE, et al. Decision analysis: effects of prophylactic mastectomy and oophorectomy on life expectancy among women with BRCA1 or BRCA2 mutations. N Engl J Med 1997;336: 1465-71.

17 College of Physicians and Surgeons. Guideline 616: Ovarian conservation. Manitoba: College of Physicians and Surgeons, 1999.

18 Goldstein MK, Tsevat J. Applying utility assessment at the bedside. In Chapman GB, Sonnenberg FA, eds. Decision making in health care: theory, psychology and application. Cambridge: Cambridge University Press, 2000: 313-33.

19 Stiggelbout AM. Assessing patients' preferences. In: Chapman GB, Sonnenberg FA, eds. Decision making in health care: theory, psychology and application. Cambridge: Cambridge University Press, 2000: 289-312.

20 Cher DJ, Miyamoto J, Lenert LA. Incorporating risk attitude into Markov-process decision models: importance for individual decision making. Med Decis Making 1997;17:340-50.

\section{COMMENTARY}

Recent years have seen increasing emphasis on the patient role in decision making and a move away from the traditional paternalistic medical model. ${ }^{1}$ In 1980 a Medline search identified 213 papers with the MESH heading of "decision making". By 2000 this had risen to 2508 papers.

In 1993 a report of a working party on appropriateness of patient care in $Q H C$ recognised the importance of effective information exchange with patients and the central importance of patient preferences in the choice of appropriate interventions - that is, appropriateness is not purely defined by technical elements of quality such as effectiveness, but also by patient values and perceptions. ${ }^{2}$ Furthermore, there has been increasing recognition that patient derived outcome measures need to be incorporated alongside, or in place of, traditional clinical or biochemically defined measures, both in research and practice. ${ }^{3-5}$ Patients' aspirations for the outcomes of their health care may differ from those of their clinicians, and increasing evidence suggests that patients' preferences and those of doctors differ. ${ }^{6}$

Internationally, the recognition of the changing role of the patient is being reflected in health policy. The UK NHS puts patient and public involvement at the core of recent health policy-for example, recognising the patient as expert in his/ her condition, particularly in chronic diseases. ${ }^{7-10}$ The unsuccessful review of health care initiated by Bill Clinton in the US also put patient and public choice firmly at the centre, albeit in a more market driven model. ${ }^{11}$ The future of high quality health care can no longer be defined purely, or even substantively, by professional providers: recognising and engaging the patient is an ethical ${ }^{12}$ and practical necessity, but to do this requires several important developments. Thus, doctors and other clinicians need ready access to evidence-based information in a form that can be clearly communicated to patients. ${ }^{13}{ }^{14}$ Furthermore, both patients and clinicians need to be supported in developing new relationships within the changing dynamic of the consultation. ${ }^{15}{ }^{16}$

The two preceding papers ${ }^{17}{ }^{18}$ come from our two separate research groups and describe the development and piloting of two computerised decision support tools. These projects were separately initiated, with very similar aims, but targeted at two quite different clinical conditions. Each set out to find a mechanism of (1) communicating risks and benefits of treatments clearly to patients who had to make a choice in the face of uncertainty; (2) incorporating patient preferences and/or values into the decision making process; and (3) bringing the evidence base to bear upon such difficult decisions (to the benefit of both the patient and the clinician).

Despite starting out with similar aims and using very similar methods of development, the final products of both teams differ in a number of important respects (table 1). Further development and evaluation of these tools will be undertaken with continuing contact between the two development teams, as there is much we can learn from each other. These are but two examples of the expanding field of decision support tools and decision aids. ${ }^{19}$ We predict that health care in the $21 \mathrm{st}$

Table 1 Comparison of the two studies

\begin{tabular}{|c|c|c|}
\hline & CGP & DARTs \\
\hline Setting/decision & $\begin{array}{l}\text { Oophorectomy at time of hysterectomy } \\
\text { One off decision } \\
\text { Long term outcomes }\end{array}$ & $\begin{array}{l}\text { Warfarin to prevent stroke in atrial fibrillation } \\
\text { Revocable decision } \\
\text { Shorter term outcomes }\end{array}$ \\
\hline Participants & Younger women (40-55 years) & Older people (men and women) \\
\hline Delivered by & Researcher/facilitator & Responsible clinician \\
\hline \multirow{5}{*}{$\begin{array}{l}\text { Value elicitation and } \\
\text { incorporating patient } \\
\text { preferences }\end{array}$} & Generic value elicitation (EuroQuol) & $\begin{array}{l}\text { Specific value elicitation for both outcomes + treatment health } \\
\text { states }\end{array}$ \\
\hline & $\begin{array}{l}\text { Standard gamble (SG) on a single generic health state plus time } \\
\text { trade off (but user can opt to choose a method they prefer from } \\
\text { "time trade off", SG or visual analogue scale) }\end{array}$ & $\begin{array}{l}\text { Standard gamble only, values derived from each patient for all } \\
\text { clinically relevant health states }\end{array}$ \\
\hline & Opt out function to population utilities & No opt out or use of population utilities \\
\hline & $\begin{array}{l}\text { Preferences for duration of HRT treatment incorporated, but value } \\
\text { of treatment state (i.e. "on HRT") not derived }\end{array}$ & $\begin{array}{l}\text { Value for treatment state derived and incorporated (i.e. "on } \\
\text { warfarin") }\end{array}$ \\
\hline & $\begin{array}{l}\text { Does not seek predisposition or final treatment preference of } \\
\text { patient } \\
\text { Patient alerted to types of task involved in advance to permit } \\
\text { prearranged opt outs }\end{array}$ & $\begin{array}{l}\text { Seeks predisposition/emerging disposition/final treatment } \\
\text { preference of patient }\end{array}$ \\
\hline $\begin{array}{l}\text { Risk assessment and } \\
\text { presentation }\end{array}$ & $\begin{array}{l}\text { Individual risk factors elicited, incorporated into model and used } \\
\text { to adjust output, but patient's individual risks of outcome events } \\
\text { not presented at point of elicitation of risk factors } \\
\text { Does not consider impact of change in other risk factors on } \\
\text { treatment decision (e.g. smoking/HT) }\end{array}$ & $\begin{array}{l}\text { Individual risk factors elicited and individual risks of stroke and } \\
\text { bleed on and off therapy made explicit to patient at the point of } \\
\text { elicitation of risk factors } \\
\text { Considers impact of change in other risk factors on treatment } \\
\text { decision (e.g. smoking/HT) }\end{array}$ \\
\hline Output & $\begin{array}{l}\text { Gives quantitative output in QALE and life expectancy } \\
\text { Output in the form of recommended treatment on basis of model } \\
\text { Effects of varying duration of HRT included as part of sensitivity } \\
\text { analysis }\end{array}$ & $\begin{array}{l}\text { Gives yes/no output of model } \\
\text { Uses model output as but one input to inform decision } \\
\text { No sensitivity analysis }\end{array}$ \\
\hline
\end{tabular}

$\mathrm{CGP}=$ clinical guidance programme; DARTs=Decision Analysis in Routine Treatment study; HRT=hormone replacement therapy; $Q A L E=q u a l i t y$ adjusted life expectancy. 
century will increasingly depend upon such supports to enhance quality of care and the responsiveness of clinical encounters with patients.

\section{Authors' affiliations}

Richard Thomson, Department of Epidemiology and Public Health, School of Health Sciences, Newcastle Medical School, Newcastle upon Tyne NE2 4HH, UK

Aileen Clarke, Health Services Research Unit, London School of Hygiene \& Tropical Medicine, London WCIE 7HT, UK

\section{REFERENCES}

1 Emanuel EJ, Emanuel LL. Four models of the physician-patient relationship. JAMA 1992;267:2221-6.

2 Anonymous. What do we mean by appropriate health care? Report of a Working Group prepared for the Director of Research and Development of the NHS Management Executive. Qual Health Care 1993;2:117-23

3 Fischer D, Stewart AL, Bloch DA, et al. Capturing the patient's view of change as a clinical outcome measure. JAMA 1999;282:1157-62.

4 Greenhalgh J, Meadows K. The effectivenes of the use of patient-based measures of health in routine practice in improving the process and outcomes of patient care: a literature review. J Eval Clin Pract 1999;5:401-16.

5 Nease R, Kneeland T, $\mathrm{O}^{\prime}$ Connor $G$, et al. Variation in patient utilities for outcomes of the management of chronic stable angina. JAMA 1995; 273: 1 185-90

6 Montgomery AA, Fahey T. How do patients' treatment preferences compare with those of clinicians? Qual Health Care 2001;10/suppl I):i39-43.
7 Department of Health. The NHS plan. London: Stationery Office, 2000.

8 Department of Health. The expert patient: a new approach to chronic disease management for the 21 st century. London: Department of Health, 2001.

9 Department of Health. Involving patients and the public in healthcare: a discussion document. London: Department of Health, 2001

10 Department of Health. Patient and public involvement in the new NHS London: Department of Health, 1999.

11 Caplan A. Clinton's health care reforms: an unstoppable momentum for change. BM 1993;307:813-4.

12 Doyal L. Informed consent: moral necessity or illusion? Qual Health Care 2001;10(suppl I):i29-33.

13 Elwyn G, Edwards A. Evidence based patient choice. Oxford: Oxford University Press, 2001.

14 Gross PA, Greenfield S, Cretin S, et al. Optimal methods for guideline implementation: conclusions from Leeds Castle meeting. Med Care 2001;8:1185-92.

15 Elwyn G, Edwards A, Kinnersley P. Shared decision-making in primary care: the neglected second half of the consultation. Br J Gen Pract 1999:49:477-82.

16 Elwyn G, Edwards A, Gwyn R, et al. Towards a feasible model for shared decision making: focus group study with general practice registrars. BN 1999;319:753-6.

17 Pell I, Dowie J, Clarke A, et al. Development and preliminary evaluation of a clinical guidance programme for the decision about prophylactic oophorectomy in women undergoing a hysterectomy. Qual Saf Health Care 2002;11:32-9.

18 Thomson R, Robinson A, Greenaway J, et al. Development and description of a decision analysis based decision support tool for stroke prevention in atrial fibrillation. Qual Saf Health Care 2002;11:25-31.

19 O'Connor AM, Rostom A, Fiset V, et al. Decision aids for patients facing health treatment or screening decisions: systematic review. BMW $1999 ; 319: 731-4$

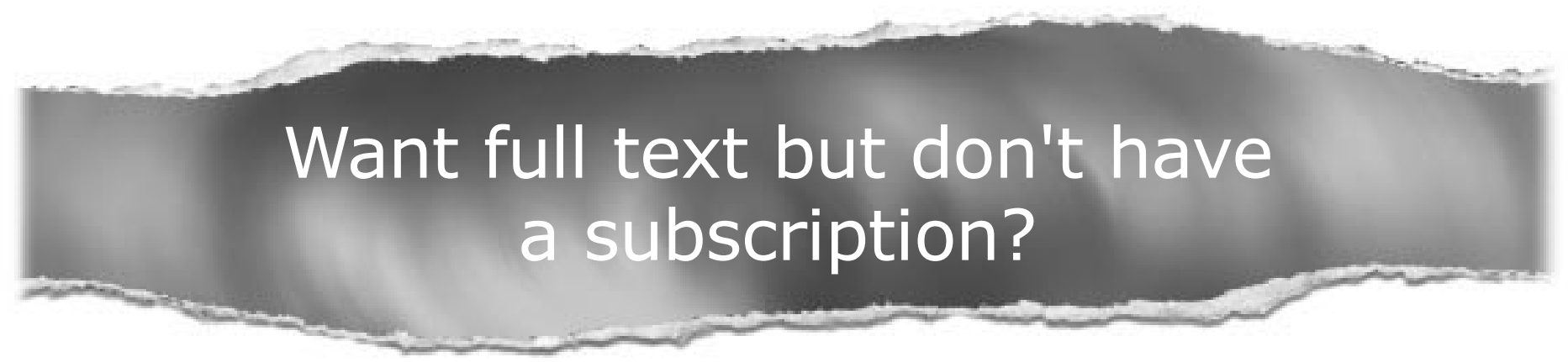

\section{Pay per view}

For just $\$ 8$ you can purchase the full text of individual articles using our secure online ordering service. You will have access to the full text of the relevant article for 48 hours during which time you may download and print the pdf file for personal use.

\section{www.qualityhealthcare.com}

